\title{
Lagrangians of Hypergraphs
}

\author{
J. M. TALBOT† \\ Merton College, Oxford OX1 4JD, UK \\ (e-mail: talbot@maths.ox.ac.uk)
}

Received 1 December 2000; revised 17 September 2001

\begin{abstract}
How large can the Lagrangian of an $r$-graph with $m$ edges be? Frankl and Füredi [1] conjectured that the $r$-graph of size $m$ formed by taking the first $m$ sets in the colex ordering of $\mathbf{N}^{(r)}$ has the largest Lagrangian of all $r$-graphs of size $m$. We prove the first 'interesting' case of this conjecture, namely that the 3-graph with $\left(\begin{array}{l}t \\ 3\end{array}\right)$ edges and largest Lagrangian is $[t]^{(3)}$. We also prove that this conjecture is true for 3-graphs of several other sizes.

For general $r$-graphs we prove a weaker result: for $t$ sufficiently large, the $r$-graph of size $\left(\begin{array}{l}t \\ r\end{array}\right)$, supported on $t+1$ vertices and with largest Lagrangian, is $[t]^{(r)}$.
\end{abstract}

\section{Introduction}

For a set $V$ let $V^{(r)}$ be the collection of all subsets of $V$ of size $r$. An $r$-uniform hypergraph, or $r$-graph, $G$ consists of a set $V$ of vertices and a set $E \subseteq V^{(r)}$ of edges. An edge $e=\left\{a_{1}, a_{2}, \ldots, a_{r}\right\}$ will be denoted by $a_{1} a_{2} \ldots a_{r}$. So, for example, if $r=3$ then 379 represents the edge $\{3,7,9\}$. For any integer $n \in \mathbf{N}$ we denote the set $\{1, \ldots, n\}$ by $[n]$. Let $K_{t}^{(r)}$ denote the complete r-graph of order $t$, that is, the $r$-graph of order $t$ containing all possible edges. Define

$$
\operatorname{ex}\left(n, K_{t}^{(r)}\right)=\max \left\{|E|: G=(V, E) \text { is a } K_{t}^{(r)} \text {-free } r \text {-graph, }|V|=n\right\}
$$

and

$$
\gamma\left(K_{t}^{(r)}\right)=\lim _{n \rightarrow \infty} \frac{\operatorname{ex}\left(n, K_{t}^{(r)}\right)}{\left(\begin{array}{l}
n \\
r
\end{array}\right)}
$$


We remind the reader of the definition of the Lagrangian of an $r$-graph. For an $r$-graph $G$ of order $n$ the weight polynomial, $w(G)$, is

$$
w(G, \mathbf{x})=\sum_{e \in E} \prod_{i \in e} x_{i} .
$$

We will call $\mathbf{x}=\left(x_{1}, \ldots, x_{n}\right) \in \mathbf{R}^{n}$ a legal weighting (for $G$ ) if

(i) $\forall i \in[n] \quad x_{i} \geqslant 0$,

(ii) $\sum_{i=1}^{n} x_{i}=1$.

The Lagrangian of $G$ is then defined to be $\lambda(G)=\max w(G, \mathbf{x})$, where the maximum is over all legal weightings for $G$. (Note that this maximum is clearly always attained.) We will call a legal weighting $\mathbf{x}$ optimal if, in addition to the above, we have

(iii) $w(G, \mathbf{x})=\lambda(G)$.

Lagrangians were introduced for 2-graphs by Motzkin and Straus in 1965 [3] when they gave a new proof of Turán's theorem. They determined the following simple expression for the Lagrangian of a 2-graph.

Theorem 1.1 (Motzkin and Straus [3]). If $G$ is a 2-graph in which a largest clique has order t, then

$$
\lambda(G)=\lambda\left(K_{t}^{(2)}\right)=\frac{1}{2}\left(1-\frac{1}{t}\right) .
$$

We leave the proof as a simple exercise for the reader unfamiliar with Lagrangians (alternatively see the proof of Lemma 2.2).

Theorem 1.2 (Turán's theorem [6, 3]).

$$
\gamma\left(K_{t}^{(2)}\right)=1-\frac{1}{t-1} .
$$

Proof. If $G$ is a $K_{t}^{(2)}$-free 2-graph of order $n$, then Theorem 1.1 implies that

$$
\lambda(G) \leqslant \frac{1}{2}\left(1-\frac{1}{t-1}\right) .
$$

Also $\lambda(G)$ is bounded below by the value of $w(G, \mathbf{x})$ given by placing weights equal to $\frac{1}{n}$ at each vertex. This gives the required upper bound for $\gamma\left(K_{t}^{(r)}\right)$. For the other direction of the inequality consider the complete $(t-1)$-partite 2 -graph on $n$ vertices formed by taking the vertex classes to be as equal as possible.

The problem of determining $\gamma\left(K_{t}^{(r)}\right)$, for $t \geqslant r \geqslant 2$, is known as Turán's problem. In contrast with the case of $r=2$, very little is known concerning Turán's problem for $r \geqslant 3$ (see, for example, Sidorenko [5]). The new proof of the 2-graph case using Lagrangians aroused interest in the study of Lagrangians for general $r$-graphs. However, as may be expected given the difficulty of Turán's problem for $r \geqslant 3$, determining the Lagrangian of a general $r$-graph is nontrivial. Indeed, the obvious generalization of Motzkin and 
Straus's result is false, and there are numerous examples of $K_{t}^{(r)}$-free $r$-graphs satisfying $\lambda(G)>\lambda\left(K_{t}^{(r)}\right)$. Moreover, there are many examples of $r$-graphs that do not achieve their Lagrangian on any proper subhypergraph.

Frankl and Füredi [1] asked the following natural question. Given $r \geqslant 3$ and $m \in \mathbf{N}$, how large can the Lagrangian of an $r$-graph with $m$ edges be? In order to state their conjecture on this problem we require the following definition. For distinct $A, B \in \mathbf{N}^{(r)}$ we say that $A$ is less than $B$ in the colex ordering if $\max (A \triangle B) \in B$. So, for example, we have $246<156$ in $\mathbf{N}^{(3)}$.

Conjecture 1.3 (Frankl and Füredi [1]). The r-graph with $m$ edges formed by taking the first $m$ sets in the colex ordering of $\mathbf{N}^{(r)}$ has the largest Lagrangian of all r-graphs with $m$ edges. In particular, the r-graph with $\left(\begin{array}{l}t \\ r\end{array}\right)$ edges and largest Lagrangian is $[t]^{(r)}$.

Theorem 1.1 trivially implies that this conjecture is true for $r=2$. However, for $r \geqslant 3$ very little was previously known (see, for example, [4]). In the next section we prove our main result (Theorem 2.1) for the 3-graph case of this conjecture. In particular, we show that the 3-graph with $\left(\begin{array}{l}t \\ 3\end{array}\right)$ edges and largest Lagrangian is $[t]^{(3)}$. We also discuss those cases of Conjecture 1.3 for 3-graphs not dealt with by Theorem 2.1. In the final section we give a weaker result for $r \geqslant 4$, showing, in particular, that for $t$ sufficiently large, the $r$-graph of size $\left(\begin{array}{l}t \\ r\end{array}\right)$, supported on $t+1$ vertices and with largest Lagrangian, is $[t]^{(r)}$.

\section{Main result}

Theorem 2.1. Let $m$ and $t$ be integers satisfying

$$
\left(\begin{array}{l}
t \\
3
\end{array}\right) \leqslant m \leqslant\left(\begin{array}{l}
t \\
3
\end{array}\right)+\left(\begin{array}{c}
t-1 \\
2
\end{array}\right)-t
$$

Then Conjecture 1.3 is true for $r=3$ and this value of $m$. In particular, Conjecture 1.3 is true for 3-graphs with $\left(\begin{array}{l}t \\ 3\end{array}\right)$ edges.

We will denote the $r$-graph with $m$ edges formed by taking the first $m$ elements in the colex ordering of $\mathbf{N}^{(r)}$ by $C_{r, m}$.

We first need to establish the following three easy lemmas concerning simple properties of Lagrangians. They provide useful facts about any $r$-graph $G$ of size $m$ satisfying $\lambda(G)=\max \{\lambda(H): H$ is an $r$-graph of size $m\}$.

The first lemma tells us that we may assume that any such $r$-graph is covering, in the sense that any two vertices lie in at least one common edge. (Note that when $r=2$ this single lemma is enough to establish the truth of Conjecture 1.3, since a covering 2-graph is simply a complete 2 -graph.) This lemma also provides a useful way of comparing the weights of distinct vertices.

The second lemma simply says that we may assume that $G$ is left-compressed, while the third lemma implies that we need not compare $\lambda(G)$ directly with $\lambda\left(C_{r, m}\right)$. For the values of $m$ that interest us, it is sufficient to check that $\lambda(G) \leqslant \lambda\left([t]^{(r)}\right)$. The proofs of all three lemmas are immediate. 
For an $r$-graph $G=(V, E)$ we will denote the $(r-1)$-neighbourhood of a vertex $i \in V$ by $E_{i}=\left\{A \in V^{(r-1)}: A \cup\{i\} \in E\right\}$. Similarly, we will denote the $(r-2)$-neighbourhood of a pair of vertices $i, j \in V$ by $E_{i j}=\left\{B \in V^{(r-2)}: B \cup\{i, j\} \in E\right\}$. We will denote the complement of $E_{i}$ by $E_{i}^{c}=\left\{A \in V^{(r-1)}: A \cup\{i\} \in V^{(r)} \backslash E\right\}$. Similarly we define $E_{i j}^{c}=\left\{A \in V^{(r-2)}: A \cup\{i, j\} \in V^{(r)} \backslash E\right\}$.

We will impose two additional conditions on any optimal legal weighting $\mathbf{x}=\left(x_{1}, \ldots, x_{n}\right)$ for an $r$-graph $G$ :

(iv) $x_{1} \geqslant x_{2} \geqslant \cdots \geqslant x_{n} \geqslant 0$,

(v) $\left|\left\{i: x_{i}>0\right\}\right|$ is minimal, i.e., if $\mathbf{y}$ is a legal weighting for $G$ satisfying $\left|\left\{i: y_{i}>0\right\}\right|<$ $\left|\left\{i: x_{i}>0\right\}\right|$, then $w(G, \mathbf{y})<\lambda(G)$.

Lemma 2.2 (Frankl and Rödl [2]). Let $G=(V, E)$ be an r-graph and $\mathbf{x}=\left(x_{1}, \ldots, x_{n}\right)$ be an optimal legal weighting for $G$ with $k \leqslant n$ nonzero weights. Then, for every $\{i, j\} \in[k]^{(2)}$,

(a) $w\left(E_{i}, \mathbf{x}\right)=w\left(E_{j}, \mathbf{x}\right)$,

(b) there is an edge in $E$ containing both $i$ and $j$.

Proof. Suppose, for a contradiction, that there exist $\{i, j\} \in[k]^{(2)}$ with $w\left(E_{i}, \mathbf{x}\right)>w\left(E_{j}, \mathbf{x}\right)$. We define a new legal weighting $\mathbf{y}$ for $G$ as follows. Let $0<\delta \leqslant x_{j}$ and define $y_{l}=x_{l}$ for $l \neq i, j, y_{i}=x_{i}+\delta$ and $y_{j}=x_{j}-\delta$. Then $\mathbf{y}$ is clearly a legal weighting for $G$, and

$$
w(G, \mathbf{y})-w(G, \mathbf{x})=\delta\left(w\left(E_{i}, \mathbf{x}\right)-w\left(E_{j}, \mathbf{x}\right)\right)-\delta^{2} w\left(E_{i j}, \mathbf{x}\right) .
$$

For sufficiently small $\delta$ this is strictly positive, contradicting $w(G, \mathbf{x})=\lambda(G)$. Hence part (a) holds.

For part (b) suppose there exist $\{i, j\} \in[k]^{(2)}$ such that no edge in $E$ contains both $i$ and $j$. Let $\mathbf{y}$ be defined as above with $\delta=x_{j}$. Since $E_{i j}=\emptyset$, part (a) and (2.1) imply that $w(G, \mathbf{y})=w(G, \mathbf{x})=\lambda(G)$. However, $\left|\left\{i: y_{i}>0\right\}\right|=k-1$, contradicting the minimality of $k$. Hence part (b) also holds.

Let $E \subset \mathbf{N}^{(r)}, e \in E$ and $i, j \in \mathbf{N}$ with $i<j$. Then define

$$
L_{i j}(e)= \begin{cases}(e \backslash\{j\}) \cup\{i\}, & \text { if } i \notin e \text { and } j \in e, \\ e, & \text { otherwise, }\end{cases}
$$

and

$$
\mathscr{C}_{i j}(E)=\left\{L_{i j}(e): e \in E\right\} \cup\left\{e: e, L_{i j}(e) \in E\right\} .
$$

We say that $E$ is left-compressed if $\mathscr{C}_{i j}(E)=E$ for every $1 \leqslant i<j$.

Lemma 2.3. Let $G=(V, E)$ be an r-graph of order $n, i, j \in[n]$ with $i<j$ and $\mathbf{x}=$ $\left(x_{1}, \ldots, x_{n}\right)$ be an optimal legal weighting for $G$. Write $G_{i j}=\left(V, \mathscr{C}_{i j}(E)\right)$. Then

$$
w(G, \mathbf{x}) \leqslant w\left(G_{i j}, \mathbf{x}\right) .
$$


Proof. Consider the difference

$$
w\left(G_{i j}, \mathbf{x}\right)-w(G, \mathbf{x})=\sum_{\substack{e \in E, L_{i j}(e) \notin E \\ i \notin e, j \in e}} w(e \backslash\{j\}, \mathbf{x})\left(x_{i}-x_{j}\right) .
$$

This is nonnegative since $i<j$ implies that $x_{i} \geqslant x_{j}$.

Lemma 2.4. For any integers $m, t$ and $r$ satisfying

$$
\left(\begin{array}{l}
t \\
r
\end{array}\right) \leqslant m \leqslant\left(\begin{array}{l}
t \\
r
\end{array}\right)+\left(\begin{array}{c}
t-1 \\
r-1
\end{array}\right)
$$

we have

$$
\lambda\left(C_{r, m}\right)=\lambda\left([t]^{(r)}\right)
$$

Proof. Firstly we note that $[t]^{(r)} \subseteq C_{r, m}$ implies that $\lambda\left(C_{r, m}\right) \geqslant \lambda\left([t]^{(r)}\right)$.

Let $\mathbf{x}=\left(x_{1}, \ldots, x_{t+1}\right)$ be an optimal legal weighting for $C_{r, m}$ using $k \leqslant t+1$ nonzero weights. As the pair of vertices $t$ and $t+1$ do not appear in a common edge of $C_{r, m}$, Lemma 2.2(b) implies that $x_{t+1}=0$. Hence $k \leqslant t$ and

$$
\lambda\left(C_{r, m}\right)=w\left(C_{r, m}, \mathbf{x}\right)=w\left([t]^{(r)}, \mathbf{x}\right) \leqslant \lambda\left([t]^{(r)}\right) .
$$

So $\lambda\left(C_{r, m}\right)=\lambda\left([t]^{(r)}\right)$.

We will now give an outline of the proof of Theorem 2.1. Let $\lambda_{m}^{r}=\max \{\lambda(G): G$ is an $r$-graph with $m$ edges $\}$. Suppose $G=(V, E)$ is a 3-graph satisfying $\lambda(G)=\lambda_{m}^{3}$ for $m=|E|$ and that

$$
\left(\begin{array}{l}
t \\
3
\end{array}\right) \leqslant m \leqslant\left(\begin{array}{l}
t \\
3
\end{array}\right)+\left(\begin{array}{c}
t-1 \\
2
\end{array}\right)-t
$$

Our proof involves a type of 'compression' on the edges of $G$. We remove certain edges from $E$ and insert others. We then need to check two conditions: firstly, that the total weight of the 3-graph (with a slightly modified weighting) has not decreased, and secondly that the number of edges we have added does not exceed the number previously removed.

Going into a little more detail, let us suppose that an optimal legal weighting for $G$ uses $k$ nonzero weights, $x_{1}, \ldots, x_{k}$. If $k \leqslant t$ then we are done (since then those edges of $G$ receiving positive weight belong to $[t]^{(3)}$ and so $\lambda(G) \leqslant \lambda\left([t]^{(3)}\right.$ and the theorem follows from Lemma 2.4). So we may suppose, for a contradiction, that $k \geqslant t+1$. Our aim is to show that most of the edges in $[k]^{(3)}$ are contained in $E$. We show that if too many edges in $[k-1]^{(3)}$ are missing from $E$ then we can remove the weight from the lightest vertex, $k$, and place it at vertex $k-1$. This reduces the weight of $G$ but also reduces the number of edges in $E$ (since any edge containing the vertex $k$ now has zero weight and so may be discarded). We may then insert new edges into $E$ (using some of the edges in $\left.[k-1]^{(3)} \backslash E\right)$ and hence produce a new 3-graph $G^{\prime}$ with the same number of edges as $G$ but with a larger Lagrangian, clearly contradicting the maximality of $\lambda(G)$. The same type of argument is then repeated but this time the weight from vertex $k-1$ is removed and added to vertex $k$. We can again construct a new 3-graph with a larger Lagrangian than 
$G$ if the number of edges in $\left([k-2]^{(2)} \times\{k\}\right) \backslash E$ is too large. Combining these two results tells us that $\left|[k]^{(3)} \backslash E\right|$ must be small. Hence, if $m$ is in the range given in the statement of Theorem 2.1, then any optimal legal weighting for $G$ can only use at most $t$ nonzero weights. So $\lambda(G) \leqslant \lambda\left([t]^{(3)}\right)$, which, as Lemma 2.4 tells us, is enough to prove the result.

Proof of Theorem 2.1. Let $G=(V, E)$ be a 3-graph with $m$ edges satisfying $\lambda(G)=\lambda_{m}^{3}$. Suppose further that there is an integer $t$ such that

$$
\left(\begin{array}{l}
t \\
3
\end{array}\right) \leqslant m \leqslant\left(\begin{array}{l}
t \\
3
\end{array}\right)+\left(\begin{array}{c}
t-1 \\
2
\end{array}\right)-t
$$

Let $\mathbf{x}=\left(x_{1}, \ldots, x_{n}\right)$ be an optimal legal weighting for $G$ that uses exactly $k$ nonzero weights (i.e., $x_{1} \geqslant \cdots \geqslant x_{k}>x_{k+1}=\cdots=x_{n}=0$ ). We will show that the number of edges in $G$ must satisfy

$$
|E| \geqslant\left(\begin{array}{c}
k-1 \\
3
\end{array}\right)+\left(\begin{array}{c}
k-2 \\
2
\end{array}\right)-(k-2)
$$

Hence, if

$$
\left(\begin{array}{l}
t \\
3
\end{array}\right) \leqslant m \leqslant\left(\begin{array}{l}
t \\
3
\end{array}\right)+\left(\begin{array}{c}
t-1 \\
2
\end{array}\right)-t
$$

then $k \leqslant t$ and so the Lagrangian of $G$ is achieved on $t$ vertices. Hence $\lambda(G) \leqslant \lambda\left([t]^{(3)}\right)$ and, by Lemma 2.4, Conjecture 1.3 is true for such values of $m$.

We aim to show that if $|E|$ is small compared to $k$, that is, if (2.2) does not hold, then we can find another 3-graph, $G^{\prime}$, with the same number of edges as $G$ satisfying $\lambda\left(G^{\prime}\right)>\lambda(G)$, contradicting the maximality of $\lambda(G)$.

We know, by Lemma 2.2(b), that the vertices $k-1$ and $k$ appear in some common edge $e \in E$. Also, by Lemma 2.3, we may suppose that $E$ is left-compressed and hence $1 k-1 k \in E$. (Recall that $1 k-1 k$ denotes the edge $\{1, k-1, k\}$.) Define $b=\max \{i: i k-1 k \in E\}$. Then, since $E$ is left-compressed, we have $E_{i}=\{u v: i u v \in E\}=\{1, \ldots, i-1, i+1, \ldots, k\}^{(2)}$, for $1 \leqslant i \leqslant b$. Also, by Lemma $2.2(\mathrm{a})$, we have $w\left(E_{1}, \mathbf{x}\right)=w\left(E_{2}, \mathbf{x}\right)=\cdots=w\left(E_{b}, \mathbf{x}\right)$. A simple calculation then yields $x_{1}=x_{2}=\cdots=x_{b}$. Note that, since $b>k-4$ implies that (2.2) holds, we may suppose that $b \leqslant k-4$.

The following three lemmas will provide the lower bound on $|E|$, proving (2.2). In particular, Lemma 2.5 implies that $E$ contains most of the first $\left(\begin{array}{c}k-1 \\ 3\end{array}\right)$ edges in the colex ordering of $\mathbf{N}^{(r)}$, while Lemma 2.7 implies that $E$ also contains most of the next $\left(\begin{array}{c}k-2 \\ 2\end{array}\right)$ edges.

Lemma 2.5. Let $k, b$ and $E$ be as defined in the proof of Theorem 2.1; then

$$
\left|[k-1]^{(3)} \backslash E\right| \leqslant\left\lceil b\left(1+\frac{k-(b+2)}{k-3}\right)\right\rceil .
$$

Lemma 2.6. Let $k, b$ and $E$ be as defined in the proof of Theorem 2.1; then

$$
\left|[k-2]^{(2)} \backslash E_{k-1}\right| \leqslant b .
$$


Lemma 2.7. Let $k, b$ and $E$ be as defined in the proof of Theorem 2.1; then

$$
\left|[k-2]^{(2)} \backslash E_{k}\right| \leqslant b \text {. }
$$

Once these lemmas are verified we obtain, using Lemma 2.5 and Lemma 2.7,

$$
\begin{aligned}
|E| & =\left|[k-1]^{(3)} \cap E\right|+\left|[k-2]^{(2)} \cap E_{k}\right|+\left|E_{k-1 k}\right| \\
& \geqslant\left(\begin{array}{c}
k-1 \\
3
\end{array}\right)-\left\lceil b\left(1+\frac{k-(b+2)}{k-3}\right)\right]+\left(\begin{array}{c}
k-2 \\
2
\end{array}\right)-b+b .
\end{aligned}
$$

It is then easy to check that

$$
\left\lceil b\left(1+\frac{k-(b+2)}{k-3}\right)\right\rceil \leqslant k-2,
$$

and so (2.2) holds and the theorem is proved.

We must now prove the three lemmas.

Proof of Lemma 2.5. We define a new legal weighting for $G, \mathbf{y}$, as follows. Let $y_{i}=x_{i}$ for $i \neq k-1, k, y_{k-1}=x_{k-1}+x_{k}$ and $y_{k}=0$. Clearly $\mathbf{y}=\left(y_{1}, \ldots, y_{k}\right)$ is a legal weighting for $G$.

Lemma 2.2(a) implies that $w\left(E_{k-1}, \mathbf{x}\right)=w\left(E_{k}, \mathbf{x}\right)$, so

$$
\begin{aligned}
w(G, \mathbf{y})-w(G, \mathbf{x}) & =x_{k}\left(w\left(E_{k-1}, \mathbf{x}\right)-w\left(E_{k}, \mathbf{x}\right)\right)-x_{k}^{2} \sum_{i=1}^{b} x_{i} \\
& =-b x_{1} x_{k}^{2} .
\end{aligned}
$$

Since $y_{k}=0$ we can remove all edges containing $k$ from $E$ to give a new 3-graph $\bar{G}=(V, \bar{E})$ with $w(\bar{G}, \mathbf{y})=w(G, \mathbf{y})$ and $|\bar{E}|=|E|-\left|E_{k}\right|$. We will show that if Lemma 2.5 fails to hold then there exists a set of edges $F \subseteq[k-1]^{(3)} \backslash E$ satisfying

$$
w(F, \mathbf{y})>b x_{1} x_{k}^{2}
$$

and

$$
|F| \leqslant\left|E_{k}\right| .
$$

Then, using (2.3), (2.4) and (2.5), the 3-graph $G^{\prime}=\left(V, E^{\prime}\right)$, where $E^{\prime}=\bar{E} \cup F$, satisfies $\left|E^{\prime}\right| \leqslant|E|$ and

$$
\begin{aligned}
w\left(G^{\prime}, \mathbf{y}\right) & =w(\bar{G}, \mathbf{y})+w(F, \mathbf{y}) \\
& >w(G, \mathbf{y})+b x_{1} x_{k}^{2} \\
& =w(G, \mathbf{x}) .
\end{aligned}
$$

Hence $\lambda\left(G^{\prime}\right)>\lambda(G)$, contradicting the maximality of $\lambda(G)$. Our next task is to construct the set of edges $F$.

Consider the equality given by Lemma $2.2(\mathrm{a}), w\left(E_{1}, \mathbf{x}\right)=w\left(E_{k-1}, \mathbf{x}\right)$. Since $E$ is leftcompressed this implies that

$$
x_{1}=x_{k-1}+\frac{w\left(E_{1} \cap E_{k-1}^{c}, \mathbf{x}\right)}{w\left(E_{1 k-1}, \mathbf{x}\right)} .
$$


Hence

$$
b x_{1} x_{k}^{2}=b x_{k-1} x_{k}^{2}+\frac{b x_{k}^{3} \sum_{i=b+1}^{k-2} x_{i}}{\sum_{i=2, i \neq k-1}^{k} x_{i}}+\frac{b x_{k}^{2} w(C, \mathbf{x})}{\sum_{i=2, i \neq k-1}^{k} x_{i}}
$$

where $C=[k-2]^{(2)} \backslash E_{k-1}$. Then, since $x_{1} \geqslant x_{2} \geqslant \cdots \geqslant x_{k}$,

$$
\begin{aligned}
b x_{1} x_{k}^{2} & \leqslant b x_{k-1} x_{k}^{2}+\frac{b x_{k}^{3}(k-2-b)}{k-3}+\frac{b x_{k}^{2} w(C, \mathbf{x})}{x_{k}(k-2)} \\
& \leqslant b x_{k-1} x_{k}^{2}\left(1+\frac{k-(b+2)}{k-3}\right)+\frac{b x_{k} w(C, \mathbf{x})}{k-2} .
\end{aligned}
$$

Define

$$
\alpha=\left\lceil\frac{b|C|}{k-2}\right\rceil \text { and } \beta=\left\lceil b\left(1+\frac{k-(b+2)}{k-3}\right)\right\rceil .
$$

Let the set $F_{1} \subseteq[k-1]^{(3)} \backslash E$ consist of the $\alpha$ heaviest edges in $[k-1]^{(3)} \backslash E$ containing the vertex $k-1$. Recalling that $y_{k-1}=x_{k-1}+x_{k}$, we have

$$
w\left(F_{1}, \mathbf{y}\right) \geqslant \alpha x_{k-1} x_{k}^{2}+\frac{b x_{k} w(C, \mathbf{x})}{k-2} .
$$

So, using (2.6),

$$
w\left(F_{1}, \mathbf{y}\right)-b x_{1} x_{k}^{2} \geqslant-x_{k-1} x_{k}^{2}(\beta-\alpha) .
$$

We now distinguish two cases.

\section{Case 1: $\alpha>\beta$}

In this case $w\left(F_{1}, \mathbf{y}\right)-b x_{1} x_{k}^{2}>0$, so defining $F=F_{1}$ satisfies (2.4). We need to check that (2.5) holds, i.e., that $|F| \leqslant\left|E_{k}\right|$. We have $|F|=\alpha=\left\lceil\frac{b|C|}{k-2}\right\rceil$ and, since $E$ is left-compressed, $[b]^{(2)} \cup\{1, \ldots, b\} \times\{b+1, \ldots, k-1\} \subseteq E_{k}$. Hence

$$
\left|E_{k}\right| \geqslant \frac{b(2 k-(b+3))}{2} \geqslant \frac{b(k+1)}{2} .
$$

Also, since $C \subset[k-2]^{(2)}$, we have

$$
|C| \leqslant\left(\begin{array}{c}
k-2 \\
2
\end{array}\right)
$$

So using (2.8) and (2.9) we obtain

$$
\frac{b|C|}{k-2} \leqslant \frac{b(k+1)}{2} \leqslant\left|E_{k}\right| .
$$

So both (2.4) and (2.5) are satisfied. Thus we may construct the new 3-graph $G^{\prime}=\left(V, E^{\prime}\right)$ as described above with $\left|E^{\prime}\right| \leqslant|E|$ and $\lambda\left(G^{\prime}\right)>\lambda(G)$, contradicting the maximality of $\lambda(G)$.

Case 2: $\alpha \leqslant \beta$

Suppose that Lemma 2.5 fails to hold. So $\left|[k-1]^{(3)} \backslash E\right| \geqslant \beta+1$. Let $F_{2}$ consist of any $\beta+1-\alpha$ 
edges in $[k-1]^{(3)} \backslash\left(E \cup F_{1}\right)$ and define $F=F_{1} \cup F_{2}$. Then, since $w\left(F_{2}, \mathbf{y}\right) \geqslant(\beta+1-\alpha) x_{k-1}^{3}$ and using (2.7),

$$
w(F, \mathbf{y})-b x_{1} x_{k}^{2}=w\left(F_{1}, \mathbf{y}\right)-b x_{1} x_{k}^{2}+w\left(F_{2}, \mathbf{y}\right)
$$

is strictly positive. So $F$ satisfies (2.4) and we need to check that $|F| \leqslant\left|E_{k}\right|$. This follows simply from the definition of $F$ and (2.8).

Since (2.4) and (2.5) both hold we can again construct the new 3-graph $G^{\prime}$ as described above, contradicting the maximality of $\lambda(G)$.

Proof of Lemma 2.6. This proceeds in a very similar way to the previous proof and we assume some of the notation from there.

If Lemma 2.6 fails to hold then $|C|=\left|[k-2]^{(2)} \backslash E_{k-1}\right| \geqslant b+1$. We again construct a new set of edges $F \subseteq[k-1]^{(3)} \backslash E$ and need to check that $F$ satisfies (2.4) and (2.5). Let $F$ consist of all edges in $[k-1]^{(3)} \backslash E$ containing the vertex $k-1$ (so $F=C \times\{k-1\}$ ). Then, since $y_{k-1}=x_{k-1}+x_{k}$,

$$
w(F, \mathbf{y})=\left(x_{k-1}+x_{k}\right) w(C, \mathbf{x}) \geqslant 2 x_{k} w(C, \mathbf{x}) .
$$

Using (2.6) we obtain

$$
w(F, \mathbf{y})-b x_{1} x_{k}^{2} \geqslant-b x_{k-1} x_{k}^{2}\left(1+\frac{k-(b+2)}{k-3}\right)+x_{k} w(C, \mathbf{x})\left(2-\frac{b}{k-2}\right) .
$$

In order to show that (2.4) holds it is sufficient to prove that

$$
|C|\left(2-\frac{b}{k-2}\right)>b\left(1+\frac{k-(b+2)}{k-3}\right) .
$$

This follows simply from $|C| \geqslant b+1$. To see that (2.5) holds we note that, by Lemma 2.5, we have $|F| \leqslant\left|[k-1]^{(3)} \backslash E\right| \leqslant \beta$ and in the proof of Lemma 2.5 we showed that $\beta+1 \leqslant\left|E_{k}\right|$. Hence $F$ satisfies (2.5). So, as in the proof of the previous lemma, we may construct a new 3-graph $G^{\prime}$ with the same number of edges as $G$ but with a larger Lagrangian.

Proof of Lemma 2.7. This proof is again almost identical to that of Lemma 2.5, the main difference being that this time the new legal weighting for $G$ is given by moving weight from vertex $k-1$ to vertex $k$.

Consider a new legal weighting for $G, \mathbf{z}=\left(z_{1}, \ldots, z_{k}\right)$, given by $z_{i}=x_{i}$ for $i \neq$ $k-1, k, z_{k-1}=0$ and $z_{k}=x_{k-1}+x_{k}$. By Lemma 2.2(a) $w\left(E_{k-1}, \mathbf{x}\right)=w\left(E_{k}, \mathbf{x}\right)$, so

$$
\begin{aligned}
w(G, \mathbf{z})-w(G, \mathbf{x}) & =x_{k-1}\left(w\left(E_{k}, \mathbf{x}\right)-w\left(E_{k-1}, \mathbf{x}\right)\right)-x_{k-1}^{2} \sum_{i=1}^{b} x_{i} \\
& =-b x_{1} x_{k-1}^{2} .
\end{aligned}
$$

Since $z_{k-1}=0$ we may remove all edges containing $k-1$ from $E$ to give a new 3-graph $G^{*}=\left(V, E^{*}\right)$ with $w\left(G^{*}, \mathbf{z}\right)=w(G, \mathbf{z})$ and $\left|E^{*}\right|=|E|-\left|E_{k-1}\right|$. By Lemma 2.6 we know that

$$
\left|E_{k-1}\right| \geqslant\left(\begin{array}{c}
k-2 \\
2
\end{array}\right) \text {. }
$$


We will show that if Lemma 2.7 fails to hold then there exists a set of edges $H \subseteq$ $\{1, \ldots, k-2, k\}^{(3)} \backslash E$ satisfying

$$
w(H, \mathbf{z})>b x_{1} x_{k-1}^{2}
$$

and

$$
|H| \leqslant\left(\begin{array}{c}
k-2 \\
2
\end{array}\right)
$$

Then, using (2.10), (2.11) and (2.12), the graph $G^{\prime \prime}=\left(V, E^{\prime \prime}\right)$, where $E^{\prime \prime}=E^{*} \cup H$, satisfies $\left|E^{\prime \prime}\right| \leqslant|E|$ and $\lambda\left(G^{\prime \prime}\right) \geqslant w\left(G^{\prime \prime}, \mathbf{z}\right)>\lambda(G)$, contradicting the maximality of $\lambda(G)$. We must now construct the set of edges $H$.

Consider the equality given by Lemma $2.2(\mathrm{a}), w\left(E_{1}, \mathbf{x}\right)=w\left(E_{k}, \mathbf{x}\right)$. Since $E$ is leftcompressed this implies that

$$
x_{1}=x_{k}+\frac{w\left(E_{1} \cap E_{k}^{c}, \mathbf{x}\right)}{w\left(E_{1 k}, \mathbf{x}\right)}
$$

Hence

$$
b x_{1} x_{k-1}^{2}=b x_{k-1}^{2} x_{k}+\frac{b x_{k-1}^{3} \sum_{i=b+1}^{k-2} x_{i}}{\sum_{i=2}^{k-1} x_{i}}+\frac{b x_{k-1}^{2} w(D, \mathbf{x})}{\sum_{i=2}^{k-1} x_{i}},
$$

where $D=[k-2]^{(2)} \backslash E_{k}$. Then, since $x_{1} \geqslant x_{2} \geqslant \cdots \geqslant x_{k}$,

$$
b x_{1} x_{k-1}^{2} \leqslant b x_{k-1}^{2} x_{k}+\frac{b x_{k-1}^{3}(k-(b+2))}{k-3}+\frac{b x_{k-1} w(D, \mathbf{x})}{k-2} .
$$

Let $H$ consist of those edges in $\{1, \ldots, k-2, k\}^{(3)} \backslash E$ containing the vertex $k$. So $w(H, \mathbf{z})=$ $\left(x_{k-1}+x_{k}\right) w(D, \mathbf{x})$. Suppose now that Lemma 2.7 fails to hold. So $|D| \geqslant b+1$. Using (2.13) we obtain

$$
w(H, \mathbf{z})-b x_{1} x_{k-1}^{2} \geqslant x_{k} x_{k-1}^{2}+|D| x_{k-1}^{3}\left(1-\frac{b}{k-2}\right)-\frac{b x_{k-1}^{3}(k-(b+2))}{k-3} .
$$

Then, since $|D|\left(1-\frac{b}{k-2}\right) \geqslant \frac{b(k-(b+2))}{k-3}$, we have $w(H, \mathbf{z})>b x_{1} x_{k-1}^{2}$ and so (2.11) holds. Finally, $D \subset[k-2]^{(2)}$ implies that $|H|=|D| \leqslant\left(\begin{array}{c}k-2 \\ 2\end{array}\right)$ and hence (2.12) holds. Therefore we may construct the 3-graph $G^{\prime \prime}$ as described above, contradicting the maximality of $\lambda(G)$.

Despite the fact that Theorem 2.1 deals with the very natural case of $m=\left(\begin{array}{l}t \\ 3\end{array}\right)$, the remaining values of $m$ for which Theorem 2.1 does not apply include what we feel are perhaps the most interesting cases of the problem. If $m$ satisfies

$$
\left(\begin{array}{l}
t \\
3
\end{array}\right)+\left(\begin{array}{c}
t-1 \\
2
\end{array}\right)+1 \leqslant m \leqslant\left(\begin{array}{c}
t+1 \\
3
\end{array}\right)-1,
$$

then $\lambda\left(C_{3, m}\right)>\lambda\left([t]^{(3)}\right)$. Indeed if Conjecture 1.3 is true for all 3-graphs then $\lambda_{m}^{3}$ jumps at each $m$ in the range given above. In fact Theorem 2.1 trivially implies that Conjecture 1.3 
is true in two such cases, namely,

$$
m=\left(\begin{array}{l}
t \\
3
\end{array}\right)-1 \quad \text { and } \quad m=\left(\begin{array}{l}
t \\
3
\end{array}\right)-2 .
$$

This follows from the proof of Theorem 2.1, by noting that $m \leqslant\left(\begin{array}{l}t \\ 3\end{array}\right)$ implies $E \subseteq[t]^{(3)}$ and then recalling that we may suppose that $E$ is left-compressed.

For the remaining values of $m$ we have the following approximate result. This tells us that any counterexample to Conjecture 1.3 for 3 -graphs cannot differ greatly from $C_{3, m}$.

Theorem 2.8. Let $m, t$ and $a$ satisfy $-(t-1) \leqslant a \leqslant(t-4)$ and

$$
m=\left(\begin{array}{l}
t \\
3
\end{array}\right)+\left(\begin{array}{c}
t-1 \\
2
\end{array}\right)+a .
$$

Suppose $G=(V, E)$ is a 3-graph with $m$ edges satisfying $\lambda(G)=\lambda_{m}^{3}$ and that $|V|$ is minimal in the sense that any other 3-graph $H$ satisfying $\lambda(H)=\lambda_{m}^{3}$ has at least $|V|$ vertices. Then $G$ and $C_{3, m}$ differ in at most $2(t-a-1)$ edges, i.e., $\left|E \triangle E\left(C_{3, m}\right)\right| \leqslant 2(t-a-1)$.

Proof. This follows simply from noting that the proof of Theorem 2.1 implies that $E \subset[t+1]^{(3)}$.

\section{A result for general $r$-graphs}

We have also considered Conjecture 1.3 for $r>3$. For such values of $r$, indeed for simply the next case of $r=4$, it seems very difficult to generalize the ideas used in the proof of Theorem 2.1.

The main argument used to prove Theorem 2.1 requires two conditions to be satisfied. Firstly, there must exist edges, not already present in the $r$-graph, which are 'reasonably heavy'. Secondly, the number of these edges we need to insert must not exceed the number of edges previously removed. The proof of Theorem 2.1 can be adapted for $r \geqslant 4$ so that the former condition holds. However, the latter condition has so far escaped our attempts at verification, although there is no obvious reason why it should fail.

Frankl and Füredi [1] originally asked how large the Lagrangian of an $r$-graph of order $n$ and size $m$ can be, where $m \leqslant\left(\begin{array}{l}n \\ r\end{array}\right)$. Define

$$
\lambda(n, r, m)=\max \{\lambda(G): G=(V, E) \text { is an } r \text {-graph, }|V|=n,|E|=m\} .
$$

If Conjecture 1.3 is true for given values of $r$ and $m$ then clearly $\lambda(n, r, m)=\lambda(l, r, m)$ whenever $n$ and $l$ satisfy $m \leqslant\left(\begin{array}{l}l \\ r\end{array}\right) \leqslant\left(\begin{array}{l}n \\ r\end{array}\right)$. (In other words it does not matter how many vertices we are allowed to use: the $r$-graph with $m$ edges and largest Lagrangian uses the smallest number of vertices possible.) Given that we have been unable to verify Conjecture 1.3 for any values of $m$ with $r \geqslant 4$, the following weaker result may be of interest.

Theorem 3.1. For any $r \geqslant 4$ there exist constants $\gamma_{r}$ and $t_{0}(r)$ such that, if $m$ satisfies

$$
\left(\begin{array}{l}
t \\
r
\end{array}\right) \leqslant m \leqslant\left(\begin{array}{l}
t \\
r
\end{array}\right)+\left(\begin{array}{c}
t-1 \\
r-1
\end{array}\right)-\gamma_{r} t^{r-2}
$$


with $t \geqslant t_{0}(r)$, then $\lambda(t+1, r, m)=\lambda\left(C_{r, m}\right)$. In particular, the $r$-graph supported on $t+1$ vertices with $\left(\begin{array}{l}t \\ r\end{array}\right)$ edges and largest Lagrangian is $[t]^{(r)}$.

Proof. A proof of this result follows along similar lines to the proof of Theorem 2.1 although the details are rather more involved.

We will take $\gamma_{r}=2^{2^{r}}$. This is not a best possible constant, simply a convenient value. Suppose $m$ satisfies

$$
\left(\begin{array}{l}
t \\
r
\end{array}\right) \leqslant m \leqslant\left(\begin{array}{l}
t \\
r
\end{array}\right)+\left(\begin{array}{l}
t-1 \\
r-1
\end{array}\right)-\gamma_{r} t^{r-2}
$$

and let $G=(V, E)$ be an $r$-graph of order $t+1$ and size $m$ satisfying $\lambda(G)=\lambda(t+1, r, m)$. Let $\mathbf{x}=\left(x_{1}, \ldots, x_{t+1}\right)$ be an optimal legal weighting for $G$ with $k$ nonzero weights, where $1 \leqslant k \leqslant t+1$. If $k<t+1$ then $\lambda(G) \leqslant \lambda\left([t]^{(r)}\right)$, so by Lemma 2.4 there is nothing to prove. Therefore we may suppose, for a contradiction, that $k=t+1$.

We know, by Lemma 2.2(b), that the vertices $k-1$ and $k$ appear in some common edge $e \in E$. Also, by Lemma 2.3, we may suppose that $E$ is left-compressed.

Our aim is to show that $G$ must contain more than $m$ edges, a contradiction. In order to achieve this we need to generalize Lemmas 2.5 and 2.7. First we require two rather technical lemmas.

Lemma 3.2. Let $D_{k-i}=[k-(i+1)]^{(r-1)} \cap E_{k-i}^{c}$, for $i \in[k-1]$, and $D_{k}=[k-2]^{(r-1)} \cap E_{k}^{c}$. Then, for any $j<k-i$,

$$
x_{j} \leqslant \frac{w\left(D_{k-i}, \mathbf{x}\right)}{w\left(E_{j k-i}, \mathbf{x}\right)}+\sum_{l=k-i}^{k} x_{l},
$$

and

$$
x_{k-1} \leqslant \frac{w\left(D_{k}, \mathbf{x}\right)}{w\left(E_{k-1 k}, \mathbf{x}\right)}+x_{k}
$$

Proof. We will prove the first part of this lemma; the second part follows identically. Suppose $i \in[k-1]$ and $j<k-i$; then by Lemma 2.2(a) we know that $w\left(E_{j}, \mathbf{x}\right)=w\left(E_{k-i}, \mathbf{x}\right)$. Since $E$ is left-compressed, we have

$$
\begin{aligned}
x_{j} & =x_{k-i}+\frac{w\left(E_{j} \cap E_{k-i}^{c}, \mathbf{x}\right)}{w\left(E_{j k-i}, \mathbf{x}\right)} \\
& \leqslant x_{k-i}+\frac{\sum_{l=k-i+1}^{k} x_{l} w\left(E_{j l} \cap E_{k-i l}^{c}, \mathbf{x}\right)}{w\left(E_{j k-i}, \mathbf{x}\right)}+\frac{w\left(D_{k-i}, \mathbf{x}\right)}{w\left(E_{j k-i}, \mathbf{x}\right)} \\
& \leqslant \frac{w\left(D_{k-i}, \mathbf{x}\right)}{w\left(E_{j k-i}, \mathbf{x}\right)}+\sum_{l=k-i}^{k} x_{l} .
\end{aligned}
$$

The first inequality follows from expanding $w\left(E_{j} \cap E_{k-i}^{c}, \mathbf{x}\right)$. The second inequality follows from $w\left(E_{j l} \cap E_{k-i l}^{c}, \mathbf{x}\right) \leqslant w\left(E_{j k-i}, \mathbf{x}\right)$ for $k-i+1 \leqslant l \leqslant k$. 
Note that, whenever the lower limit of a sum or product is greater than the upper limit, we take this to be the empty sum or product. These are defined to be equal to zero and one, respectively.

Lemma 3.3. For $0 \leqslant q \leqslant r-3$ and $i<j<k-d_{q}<\cdots<k-d_{1} \leqslant k-2$, we have

$$
\sum_{\substack{i_{1} \ldots i_{r-2} \in E_{k-1 k} \\ i_{q+1}<j}} x_{k-1} x_{k} \prod_{m=q+2}^{r-2} x_{i_{m}} \prod_{p=1}^{q} x_{k-d_{p}} \leqslant x_{j} w\left(E_{i j}, \mathbf{x}\right) .
$$

Proof. Let $A=\left\{i_{1}, \ldots, i_{r-2}\right\} \in E_{k-1 k}$ with $i_{1}<i_{2}<\cdots<i_{r-2}$ and $i_{q+1}<j$. For each such set $A$ we need to find a unique set $B \in E_{i j}$ such that the contribution of $A$ to the left-hand side of (3.2) is less than or equal to the contribution of $B$ to the right-hand side. The contribution of $A$ is always

$$
x_{k-1} x_{k} \prod_{m=q+2}^{r-2} x_{i_{m}} \prod_{p=1}^{q} x_{k-d_{p}} .
$$

If $A \cap\{i, j\}=\emptyset$ then $A \in E_{i j}$, so let $B=A$. Then the contribution of $B$ to (3.2) is

$$
x_{j} \prod_{m=1}^{r-2} x_{i_{m}}
$$

and since

$$
x_{k-1} x_{k} \prod_{p=1}^{q} x_{k-d_{p}} \leqslant x_{j} \prod_{m=1}^{q+1} x_{i_{m}}
$$

these terms satisfy (3.2).

If $i \in A$ but $j \notin A$ then there exists $1 \leqslant s \leqslant r-2$ such that $i=i_{s}$. Let $B=(A \cup\{k\}) \backslash\{i\}$; then $B \in E_{i j}$ and $k \in B$, but $k-1 \notin B$. This time the contribution of $B$ to (3.2) is

$$
x_{j} x_{k} \prod_{m=1, m \neq s}^{r-2} x_{i_{m}} .
$$

We have two cases. If $s \leqslant q+1$ then

$$
x_{k-1} \prod_{p=1}^{q} x_{k-d_{p}} \leqslant x_{j} \prod_{m=1, m \neq s}^{q+1} x_{i_{m}},
$$

so (3.2) is satisfied. Otherwise we have $s \geqslant q+2$ so $x_{i_{s}} \leqslant x_{i_{q+1}}$ and

$$
x_{k-1} \prod_{p=1}^{q} x_{k-d_{p}} \leqslant x_{j} \prod_{m=1}^{q} x_{i_{m}} .
$$

Hence (3.2) is satisfied.

If $j \in A$ but $i \notin A$, then there exists $q+2 \leqslant s \leqslant r-2$ such that $j=i_{s}$. Let $B=(A \cup\{k-1\}) \backslash\{j\}$; then $B \in E_{i j}$ and $k-1 \in B$ but $k \notin B$. This time the contribution 
of $B$ to $(3.2)$ is

$$
x_{j} x_{k-1} \prod_{m=1, m \neq s}^{r-2} x_{i_{m}} .
$$

Then, since

$$
x_{k} \prod_{p=1}^{q} x_{k-d_{p}} \leqslant x_{j} \prod_{m=1}^{q} x_{i_{m}}
$$

and $x_{i_{s}} \leqslant x_{i_{q+1}}$, (3.2) is satisfied.

Finally, if $i, j \in A$ then there exist $1 \leqslant s<v \leqslant r-2$ such that $i=i_{s}$ and $j=i_{v}$. Let $B=(A \cup\{k-1, k\}) \backslash\{i, j\}$; then $B \in E_{i j}$ and $k-1, k \in B$. This time the contribution of $B$ to $(3.2)$ is

$$
x_{j} x_{k-1} x_{k} \prod_{m=1, m \neq s, v}^{r-2} x_{i_{m}} .
$$

We know that $v \geqslant q+2$ but we must distinguish two cases depending on the value of $s$. First suppose that $s \leqslant q+1$; then

$$
x_{i_{v}} \prod_{p=1}^{q} x_{k-d_{p}} \leqslant x_{j} \prod_{m=1, m \neq s}^{q+1} x_{i_{m}}
$$

implies that (3.2) holds. Now suppose $s \geqslant q+2$; then

$$
x_{i_{v}} x_{i_{s}} \prod_{p=1}^{q} x_{k-d_{p}} \leqslant x_{j} \prod_{m=1}^{q+1} x_{i_{m}},
$$

so (3.2) holds in this case also.

Note that in each case the set $B$ is unique. (We can see this by considering whether or not $k-1$ and $k$ belong to $B$ in each case.) This completes the proof of the lemma.

Let $c_{q}$ and $d_{q}$ be defined as follows for $q \geqslant 0$ :

$$
\begin{array}{ll}
c_{0}=1, & c_{q+1}=\sum_{i=1}^{c_{q}}\left(d_{q}+i+1\right), \\
d_{0}=1, & d_{q+1}=c_{q}+d_{q} .
\end{array}
$$

We have the following generalizations of Lemmas 2.5 and 2.7.

Lemma 3.4. Let $k$ and $E$ be as defined in the proof of Theorem 3.1 and $c_{q}$ and $d_{q}$ be as defined above; then

$$
\left|\left[k-\left(d_{r-2}+1\right)\right]^{(r)} \backslash E\right| \leqslant c_{r-2}\left|E_{k-1 k}\right| .
$$

Lemma 3.5. Let $k$ and $E$ be as defined in the proof of Theorem 3.1 and $c_{q}$ and $d_{q}$ be as defined above; then

$$
\left|\left[k-\left(d_{r-2}+1\right)\right]^{(r-1)} \backslash E_{k}\right| \leqslant c_{r-2}\left|E_{k-1 k}\right| .
$$


Once these lemmas are verified we may complete the proof of Theorem 3.1 as follows. Since $\left|E_{k-1 k}\right|<\left(\begin{array}{c}k-2 \\ r-2\end{array}\right)$ and $E$ is left-compressed we may use Lemmas 3.4 and 3.5 to give the following lower bound for $|E|$ :

$$
|E|>\left(\begin{array}{c}
k-\left(d_{r-2}+1\right) \\
r
\end{array}\right)+\left(d_{r-2}+1\right)\left(\begin{array}{c}
k-\left(d_{r-2}+1\right) \\
r-1
\end{array}\right)-\left(d_{r-2}+2\right) c_{r-2}\left(\begin{array}{l}
k-2 \\
r-2
\end{array}\right) .
$$

Using $d_{r}<2 c_{r-1}, c_{r}<2^{2^{r}}$ and $k=t+1$, a tedious but easy calculation yields

$$
|E|>\left(\begin{array}{l}
t \\
r
\end{array}\right)+\left(\begin{array}{l}
t-1 \\
r-1
\end{array}\right)-\gamma_{r} t^{r-2}
$$

for sufficiently large $t$. This contradicts our assumption that $|E|$ lies in the range given by (3.1) and so completes the proof of Theorem 3.1.

Proof of Lemma 3.4. As in Lemma 3.2, let $D_{k-i}=[k-(i+1)]^{(r-1)} \cap E_{k-i}^{c}$, for $i \in[k-1]$, and $D_{k}=[k-2]^{(r-1)} \cap E_{k}^{c}$. We claim that the following inequality holds for every $0 \leqslant q \leqslant r-2$ :

$$
\begin{aligned}
x_{k-1} x_{k} w\left(E_{k-1 k}, \mathbf{x}\right) \leqslant \sum_{i=2}^{d_{q}} x_{k-i} w\left(D_{k-i}, \mathbf{x}\right)+ & \\
& \sum_{i_{1} \ldots i_{r-2} \in E_{k-1 k}} c_{q} x_{k-1} x_{k} \prod_{m=q+1}^{r-2} x_{i_{m}} \prod_{p=1}^{q} x_{k-d_{p}} .
\end{aligned}
$$

We prove this by induction on $q$. Since $c_{0}=1$ and $d_{0}=1$ (3.3) holds for $q=0$ with a simple equality. Now let us suppose (3.3) holds for some $0 \leqslant q \leqslant r-3$, we will show that it also holds for $q+1$. Let $l$ satisfy $d_{q}+1 \leqslant l \leqslant d_{q+1}$. Consider $i_{q+1} \in[k]$. If $k-l \leqslant i_{q+1} \leqslant k$ then $x_{i_{q+1}} \leqslant x_{k-l}$. Otherwise $i_{q+1}<k-l$, so Lemma 3.2 implies that

$$
x_{i_{q+1}} \leqslant \frac{w\left(D_{k-l}, \mathbf{x}\right)}{w\left(E_{i_{q+1} k-l}, \mathbf{x}\right)}+\sum_{j=k-l}^{k} x_{j} .
$$

Hence

$$
\begin{aligned}
\sum_{i_{1} \ldots i_{r-2} \in E_{k-1 k}} \prod_{m=q+1}^{r-2} x_{i_{m}} \leqslant \sum_{\substack{i_{1} \ldots i_{r-2} \in E_{k-1 k} \\
i_{q+1} \geqslant k-l}} x_{k-l} \prod_{m=q+2}^{r-2} x_{i_{m}}+ \\
\sum_{\substack{i_{1} \ldots i_{r-2} \in E_{k-1 k} \\
i_{q+1}<k-l}}\left(\frac{w\left(D_{k-l}, \mathbf{x}\right)}{w\left(E_{i_{q+1} k-l}, \mathbf{x}\right)}+(l+1) x_{k-l}\right) \prod_{m=q+2}^{r-2} x_{i_{m}} .
\end{aligned}
$$

So

$$
\begin{aligned}
\sum_{i_{1} \ldots i_{r-2} \in E_{k-1 k}} \prod_{m=q+1}^{r-2} x_{i_{m}} \leqslant \sum_{i_{1} \ldots i_{r-2} \in E_{k-1 k}}(l+1) x_{k-l} \prod_{m=q+2}^{r-2} x_{i_{m}}+ \\
\sum_{\substack{i_{1} \ldots i_{r-2} \in E_{k-1 k} \\
i_{q+1}<k-l}} \frac{w\left(D_{k-l}, \mathbf{x}\right) \prod_{m=q+2}^{r-2} x_{i_{m}}}{w\left(E_{i_{q+1} k-l}, \mathbf{x}\right)} .
\end{aligned}
$$


Also, by Lemma 3.3, we have

$$
\sum_{\substack{i_{1} \ldots i_{r-2} \in E_{k-1 k} \\ i_{q+1}<k-l}} \frac{x_{k-1} x_{k} \prod_{m=q+2}^{r-2} x_{i_{m}} \prod_{p=1}^{q} x_{k-d_{p}}}{w\left(E_{i_{q+1} k-l}, \mathbf{x}\right)} \leqslant x_{k-l}
$$

Using (3.4) and (3.5) we obtain

$$
\begin{aligned}
\sum_{i_{1} \ldots i_{r-2} \in E_{k-1 k}} x_{k-1} x_{k} \prod_{m=q+1}^{r-2} x_{i_{m}} \prod_{p=1}^{q} x_{k-d_{p}} \leqslant x_{k-l} w\left(D_{k-l}, \mathbf{x}\right)+ & \\
& \sum_{i_{1} \ldots i_{r-2} \in E_{k-1 k}}(l+1) x_{k-l} x_{k-1} x_{k} \prod_{m=q+2}^{r-2} x_{i_{m}} \prod_{p=1}^{q} x_{k-d_{p} .} .
\end{aligned}
$$

This last inequality holds for each $l$ satisfying $d_{q}+1 \leqslant l \leqslant d_{q+1}$.

Finally, since $d_{q+1}=c_{q}+d_{q}, c_{q+1}=\sum_{i=1}^{c_{q}}\left(d_{q}+i+1\right)$ and $x_{k-l} \leqslant x_{k-d_{q+1}}$, we can sum (3.6) over all values of $l$ with $d_{q}+1 \leqslant l \leqslant d_{q+1}$ to obtain

$$
\begin{aligned}
\sum_{i_{1} \ldots i_{r-2} \in E_{k-1 k}} c_{q} x_{k-1} x_{k} \prod_{m=q+1}^{r-2} x_{i_{m}} \prod_{p=1}^{q} x_{k-d_{p}} \leqslant & \sum_{l=d_{q}+1}^{d_{q+1}} x_{k-l} w\left(D_{k-l}, \mathbf{x}\right)+ \\
& \sum_{i_{1} \ldots i_{r-2} \in E_{k-1 k}} c_{q+1} x_{k-1} x_{k} \prod_{m=q+2}^{r-2} x_{i_{m}} \prod_{p=1}^{q+1} x_{k-d_{p} .}
\end{aligned}
$$

Hence (3.3) holds for $q+1$ and the induction is complete.

Setting $q=r-2$ in (3.3) we obtain

$$
x_{k-1} x_{k} w\left(E_{k-1 k}, \mathbf{x}\right) \leqslant \sum_{i=2}^{d_{r-2}} x_{k-i} w\left(D_{k-i}, \mathbf{x}\right)+c_{r-2} x_{k-1} x_{k}\left|E_{k-1 k}\right| \prod_{p=1}^{r-2} x_{k-d_{p}} .
$$

Now suppose that Lemma 3.4 fails to hold. Then we may proceed as in the proof of Lemma 2.5 to give a new weighting, $\mathbf{y}$, for our $r$-graph $G$ by moving the weight from vertex $k$ to vertex $k-1$. Let $y_{i}=x_{i}$, for $i \neq k-1, k, y_{k-1}=x_{k-1}+x_{k}$ and $y_{k}=0$. Clearly $\mathbf{y}$ is a legal weighting for $G$. Let $G^{\prime}$ be formed from $G$ by removing all of the edges in $G$ containing $k$. Then $w\left(G^{\prime}, \mathbf{y}\right)=w(G, \mathbf{y})=w(G, \mathbf{x})-x_{k}^{2} w\left(E_{k-1 k}, \mathbf{x}\right)$. Let $G^{*}=[k-1]^{(r)}$. Note that $G^{*}$ cannot contain more edges than $G$. Then, since we are assuming that Lemma 3.4 fails to hold,

$$
\begin{aligned}
w\left(G^{*}, \mathbf{y}\right) & =w\left(G^{\prime}, \mathbf{y}\right)+\sum_{i=1}^{d_{r-2}} x_{k-i} w\left(D_{k-i}, \mathbf{y}\right)+w\left(\left[k-\left(d_{r-2}+1\right)\right]^{(r)} \backslash E, \mathbf{y}\right) \\
& >w\left(G^{\prime}, \mathbf{y}\right)+\sum_{i=2}^{d_{r-2}} x_{k-i} w\left(D_{k-i}, \mathbf{x}\right)+c_{r-2}\left|E_{k-1 k}\right| \prod_{j=1}^{r} x_{k-d_{r-2}-j}
\end{aligned}
$$

Finally, applying (3.7) we obtain

$$
w\left(G^{*}, \mathbf{y}\right)>w\left(G^{\prime}, \mathbf{y}\right)+x_{k-1} x_{k} w\left(E_{k-1 k}, \mathbf{x}\right),
$$


and so $\lambda\left(G^{*}\right) \geqslant w\left(G^{*}, \mathbf{y}\right)>w(G, \mathbf{x})$. This contradicts the assumption that $\lambda(t+1, r, m)=$ $\lambda(G)=w(G, \mathbf{x})$ and completes the proof of Lemma 3.4.

Proof of Lemma 3.5. This is easy given the work we have already done. We proceed as in the proof of Lemma 2.7 to give a new weighting, $\mathbf{z}$, for $G$ by moving the weight from vertex $k-1$ to vertex $k$. So $z_{i}=x_{i}$, for $i \neq k-1, k, z_{k-1}=0$ and $z_{k}=x_{k-1}+x_{k}$. Then $\mathbf{z}$ is clearly a legal weighting for $G$. Now let $G^{\prime}$ be formed from $G$ by removing all of the edges in $G$ containing $k-1$. Then $w\left(G^{\prime}, \mathbf{z}\right)=w(G, \mathbf{z})=w(G, \mathbf{x})-x_{k-1}^{2} w\left(E_{k-1 k}, \mathbf{x}\right)$.

By Lemma 3.2 we have

$$
x_{k-1} \leqslant \frac{w\left(D_{k}, \mathbf{x}\right)}{w\left(E_{k-1 k}, \mathbf{x}\right)}+x_{k}
$$

so

$$
w\left(G^{\prime}, \mathbf{z}\right) \geqslant w(G, \mathbf{x})-x_{k-1} w\left(D_{k}, \mathbf{x}\right)-x_{k-1} x_{k} w\left(E_{k-1 k}, \mathbf{x}\right) .
$$

Now let $G^{*}=\{1, \ldots, k-2, k\}^{(r)}$. As before, $G^{*}$ cannot contain more edges than $G$. Suppose, for a contradiction, that Lemma 3.5 fails to hold. Then we have (via a simple calculation similar to that used in the proof of the previous lemma)

$$
\begin{array}{r}
w\left(G^{*}, \mathbf{z}\right)>w\left(G^{\prime}, \mathbf{z}\right)+x_{k-1} w\left(D_{k}, \mathbf{x}\right)+\sum_{i=2}^{d_{r-2}} x_{k-i} w\left(D_{k-i}, \mathbf{x}\right)+ \\
c_{r-2}\left|E_{k-1 k}\right| x_{k} \prod_{j=1}^{r-1} x_{k-d_{r-2}-j} .
\end{array}
$$

So, using (3.7) and (3.8) we obtain $\lambda\left(G^{*}\right) \geqslant w\left(G^{*}, \mathbf{z}\right)>w(G, \mathbf{x})$. This contradicts our assumption that $\lambda(t+1, r, m)=\lambda(G)=w(G, \mathbf{x})$ and completes the proof of Lemma 3.5. $\square$

We can perhaps claim this last result as intuitive evidence of the truth of the FranklFüredi conjecture for $r$-graphs with $\left(\begin{array}{l}t \\ r\end{array}\right)$ edges. This is because it says essentially that, if there exists a counterexample to the conjecture, then it must use at least $t+2$ positively weighted vertices, and so there is no $r$-graph whose set of edges is 'similar' to $[t]^{(r)}$ with a larger Lagrangian. Hence a counterexample, should one exist, would contain lots of 'gaps' - this seems a little implausible.

\section{Acknowledgements}

I would like to thank Imre Leader for suggesting this problem and for many invaluable discussions.

\section{References}

[1] Frankl, P. and Füredi, Z. (1989) Extremal problems whose solutions are the blow-ups of the small Witt-designs. J. Combin. Theory Ser. A 52 129-147.

[2] Frankl, P. and Rödl, V. (1984) Hypergraphs do not jump. Combinatorica 4 149-159.

[3] Motzkin, T. S. and Straus, E. G. (1965) Maxima for graphs and a new proof of a theorem of Turán. Canad. J. Math. 17 533-540. 
[4] Sarkar, A. (1999) PhD Thesis, University of Cambridge.

[5] Sidorenko, A. F. (1995) What we know and what we do not know about Turán numbers. Graphs Combin. 11 179-199.

[6] Turán, P. (1941) On an extremal problem in graph theory. Mat. Fiz. Lapok 48 436-452. (In Hungarian.) 